\title{
Mediating Fano losses in plasmonic scatterers by tuning the dielectric environment
}

A. W. Powell, and J. M. Smith

Citation: Appl. Phys. Lett. 109, 121107 (2016); doi: 10.1063/1.4962945

View online: $\mathrm{http}: / / d x . d o i . o r g / 10.1063 / 1.4962945$

View Table of Contents: http://aip.scitation.org/toc/apl/109/12

Published by the American Institute of Physics

\section{Articles you may be interested in}

Strong coupling in molecular exciton-plasmon Au nanorod array systems

Applied Physics Letters 108, 053102 (2016); 10.1063/1.4941078

Plasmonic properties of gold nanoparticles on silicon substrates: Understanding Fano-like spectra observed in reflection

Applied Physics Letters 109, 111901 (2016); 10.1063/1.4962731

Observation of Fano line shapes arising from coupling between surface plasmon polariton and waveguide modes

Applied Physics Letters 108, 051101 (2016); 10.1063/1.4940984

Sensing with toroidal metamaterial

Applied Physics Letters 110, 121108 (2017); 10.1063/1.4978672

Trimeric metasurfaces for independent control of bright and dark modes of Fano resonances

Applied Physics Letters 108, 081109 (2016); 10.1063/1.4942644

Unconventional Fano effect based spectrally selective absorption enhancement in graphene using plasmonic core-shell nanostructures

Applied Physics Letters 109, 031909 (2016); 10.1063/1.4959553

\section{Scilight}

Sharp, quick summaries illuminating the latest physics research

\section{Sign up for FREE!}

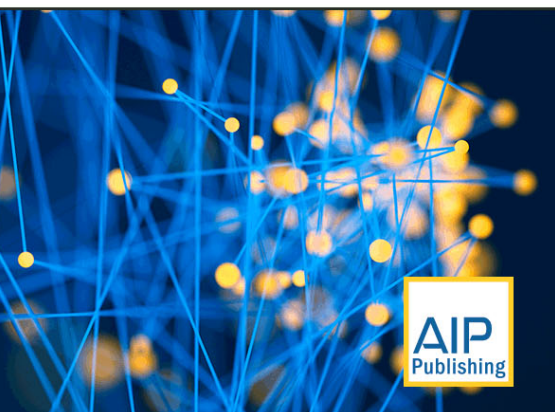




\title{
Mediating Fano losses in plasmonic scatterers by tuning the dielectric environment
}

\author{
A. W. Powell ${ }^{\text {a) }}$ and J. M. Smith ${ }^{\text {b) }}$ \\ Department of Materials, University of Oxford, Parks Road, Oxford OXI 3PH, United Kingdom
}

(Received 27 May 2016; accepted 31 August 2016; published online 20 September 2016)

\begin{abstract}
Scattering from metal nanoparticles above a high index substrate has the potential to significantly enhance light trapping in thin film devices with minimal additional cost. However, the benefits of this approach are currently limited by short wavelength losses from Fano resonances arising due to interference between the plasmon modes in a nanoparticle. In this paper, the effect of particlesubstrate interactions on the observed Fano behaviour is explored in detail, using finite-difference time-domain simulations and an analytical model. Several approaches to mitigate the Fano loss are explored, and it is found that overcoating the particle with a thin dielectric film represents by far the most effective way to remove Fano losses whilst keeping broadband forward scattering high.

Published by AIP Publishing. [http://dx.doi.org/10.1063/1.4962945]
\end{abstract}

Metal nanoparticles (NPs) and nanostructures have attracted considerable interest across a number of fields due to the strong surface plasmon resonance excited within them by incident radiation. ${ }^{1,2}$ The high charge density of noble metals leads to a very strong near field about the particle surface, which also makes them highly efficient scatterers. These properties have led to a great deal of work trying to optimise the behaviours of noble metal nanostructures to act as nanoantennas, ${ }^{3-5}$ or nanoscale sensing elements, ${ }^{6-8}$ or to enhance light trapping for thin film photovoltaics. $^{9-12}$ In each of these examples, the Fano resonances observed in the particle scattering can be extremely important. These arise due to a phase-dependant coupling between the resonant modes of a system and typically have an asymmetric spectral shape and a very narrow width compared with other plasmon resonances - this leads to an improved figure of merit for sensing applications, ${ }^{15-17}$ and can produce a greater electric field enhancement, which is of interest for nonlinear frequency generation ${ }^{18,19}$ and coupling to single emitters. ${ }^{20,21}$

The strength of the Fano resonance in a plasmonic system is dictated by the strength of the coupling between a spectrally broad, "bright" mode and a narrower, "dark" mode. ${ }^{13,14}$

Coupling between plasmon modes in a single particle can be controlled by symmetry breaking: ${ }^{15}$ the placement of a particle near a substrate has been shown to facilitate strong interaction and hybridisation between modes, ${ }^{25,26}$ and Fano resonances are very likely to be observed for particles above high index substrates, as in situations where metal particles are utilised to enhance light trapping or antireflection for photovoltaic cells. In these structures, the high optical density of states (DoS) in the substrate leads to very strong forward scattering from metal nanoparticles (NPs) above the substrate, and therefore reduced reflection across most of the spectrum. However, due to the Fano interference between modes, a phase shift occurs about any higher order resonances present, leading to a rapid switch in the direction of

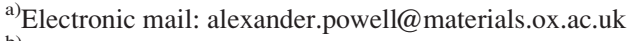

b)URL: http://www-png.materials.ox.ac.uk
}

scattering and a loss of forward coupling at shorter wavelengths (see Fig. 1). Whilst there have been attempts to describe this behaviour under specific conditions, ${ }^{23,24}$ Fano resonances remain a significant problem for light trapping devices and a rigorous discussion of the origin of this behaviour, and a mechanism for controlling it is still lacking.

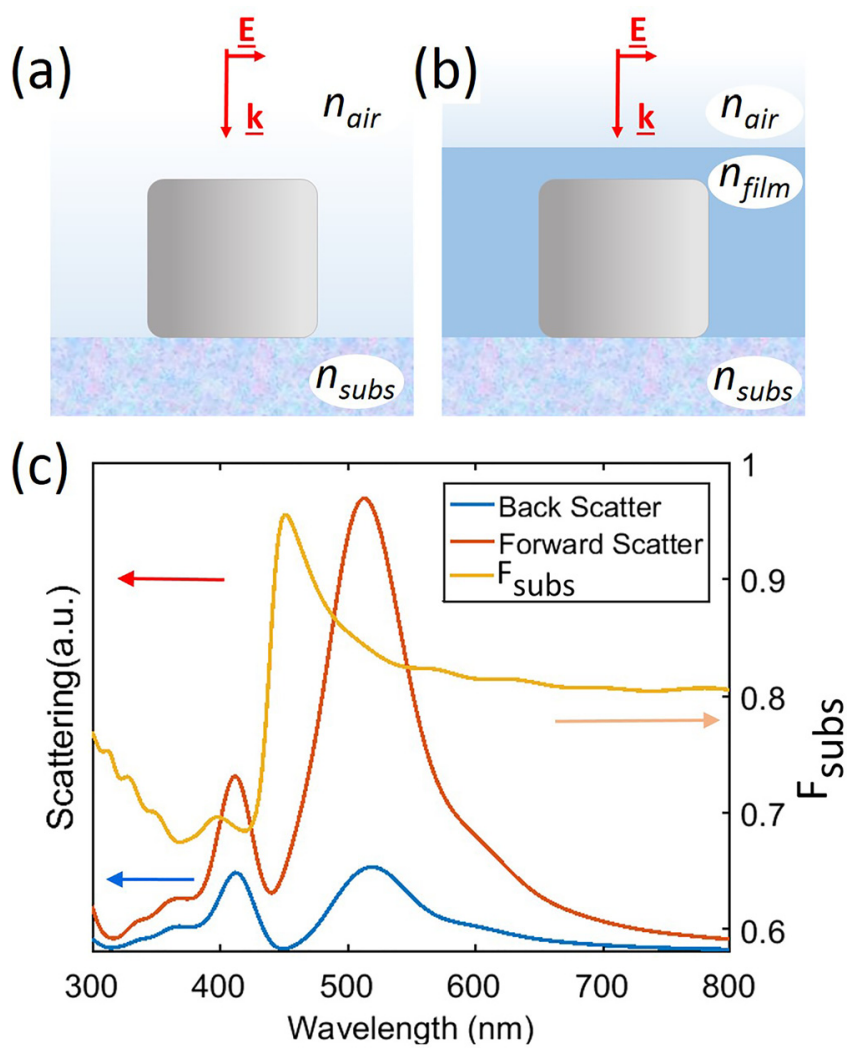

FIG. 1. (a) and (b) The simulation setup in this investigation: a single nanocube is positioned at an interface, illuminated with a normally incident plane wave and the scattering measured. In some cases, a thin dielectric film is added above the NP with a refractive index $\mathrm{n}_{\text {fim }}$, or a very thin spacer placed between the particle and the substrate with a refractive index $\mathrm{n}_{\text {spac }}$. (c) Forward and reverse scattering from a cubic Ag NP with a $75 \mathrm{~nm}$ width and $5 \mathrm{~nm}$ corner radius of curvature (RoC) above a substrate with $\mathrm{n}_{\text {subs }}=1.6$. The fraction scattered into the substrate $\mathrm{F}_{\text {subs }}$ is also shown. 
In this investigation, finite-difference time-domain (FDTD) simulations are used alongside an analytical model described by Gallinet and Martin ${ }^{13}$ to investigate the effect the surrounding dielectric environment has on Fano resonances in scattering from metal nanoparticles. The effect of increased mode hybridisation due to substrate interactions on the forward and reverse scattering is discussed, and we show that tuning the dielectric environment around the particles allows for the enhancement or suppression of the Fano effect, although this can also have consequences for the forward scattering efficiency. Several methods to mitigate Fano losses are explored and it is demonstrated that the addition of a thin film above a high index substrate can be used to entirely remove Fano losses and whilst enhancing forward scattering across the solar spectrum.

Figures 1(a) and 1(b) displays the structure used in the simulations: A cubic $\mathrm{Ag}$ nanoparticle is placed directly above a substrate with a refractive index (RI) of $\mathrm{n}_{\text {subs }}$, and in some cases, an overcoating thin film was modelled above the NP with a RI of $\mathrm{n}_{\text {film }}$, and in others, a very thin spacer is placed between the NP and the substrate with a RI of $\mathrm{n}_{\text {spac }}$. The upper layer has an RI of $\mathrm{n}_{\text {air }}$ and is set to a value of 1 unless otherwise stated. Cubes with a width of $75 \mathrm{~nm}$ and corner radius of curvature $(\mathrm{RoC})$ of $5 \mathrm{~nm}$ are used in these simulations, to approximate some examples that are both commercially available ${ }^{33}$ and readily fabricable using top down approaches such as electron beam lithography. Planar illumination is incident from the free space side normal to the layers, and the forward and reverse scattering of the particles are measured through FDTD simulations using Lumerical. ${ }^{34}$ Further details of the simulations can be found in the supplementary material.

Figure 1(c) shows the forwards and backwards scattering spectra from a $75 \mathrm{~nm} \mathrm{Ag}$ cube resting on a substrate with a refractive index of 1.6. It can be observed that whilst the broad first order mode at $514 \mathrm{~nm}$ shows weak backwards scattering away from the substrate, as would be expected due to the large DoS, the sharper second order mode at $413 \mathrm{~nm}$ shows significantly stronger backscatter. Looking at the ratio of the light scattered forward to the total scattered power $\left(\mathrm{F}_{\text {subs }}\right.$, orange line), it is clear that near to the second order mode there is a strong asymmetric dip displaying the characteristic shape of a Fano resonance. This short-wavelength loss has been observed experimentally and in simulations for a plethora of shapes, including spheres, ${ }^{27,28}$ variously shaped disks, ${ }^{23,29,30}$ cubes, ${ }^{31}$ and nanowires ${ }^{32}$ above a variety of high index substrates.
The effect of substrate refractive index on Fano losses for forward scattering from plasmonic nanoparticles is now explored: Figure 2(a) shows the forward coupling fraction $\mathrm{F}_{\text {subs }}$ from NPs above a bare dielectric substrate with varying refractive index, $\mathrm{n}_{\text {subs. }}$. The introduction of a substrate, with $\mathrm{n}_{\text {subs }}=1.4$, raises the forward scattering compared to a free space scenario and introduces a sharp Fano peak to the red of the second order mode around $500 \mathrm{~nm}$, with a small drop to the blue. As $\mathrm{n}_{\text {subs }}$ is raised, the forward coupling is improved for most wavelengths across the spectrum, but the peak in forward scattering about the Fano resonance is reduced, whilst the drop becomes far more significant. This is especially detrimental for semiconductor devices such as silicon and GaAs with very high RIs wishing to make use of the light trapping and antireflection capacities of particle arrays on their surfaces.

The explanation for this behaviour is in the interaction between the static charges in the substrate and the charge oscillations associated with the plasmon modes of the NP. Figure 2(b) shows the backward scatter from a NP at an interface with changing $\mathrm{n}_{\text {subs. }}$. As described by Zhang et al., ${ }^{15}$ when a plasmon mode is excited in a flat particle with one face near to a high index substrate, the high charge concentration about the near face of the cube produces a mirror charge polarisation in the substrate increasing the symmetry of the system and lifting the orthogonality of the modes in the NP, allowing interactions between the free space dipolar and quadrupolar modes, which hybridize to form a bonding and antibonding variant, located on the lower and upper faces of the particle (see supplementary material). The broadband, longer wavelength mode is labelled $\mathbf{D}$ going forward, is concentrated about the near face of the NP, and is therefore redshifted strongly by the substrate RI. The short wavelength mode is labelled $\mathbf{Q}$ and is concentrated about the upper face and therefore responds significantly to changes in the RI of the upper medium and shows little change here spectrally. A significant mode splitting is observed in Fig. 2(b) as $\mathrm{n}_{\text {subs }}$ increases, indicating a strong interaction between and hybridisation of the NP modes.

The connection between the Fano dip in the $\mathrm{F}_{\text {subs }}$ plots in Fig. 2(a) and the mode hybridisation shown in Fig. 2(b) can be better understood by comparing the results of FDTD simulations with a model for Fano resonances described by Gallinet and Martin. Here, the reflection spectra of a plasmonic system with two interacting modes is described as of the product of a broad, symmetric Lorentzian mode $\sigma_{s}$ and an asymmetric Fano resonance $\sigma_{a}$ which can be written

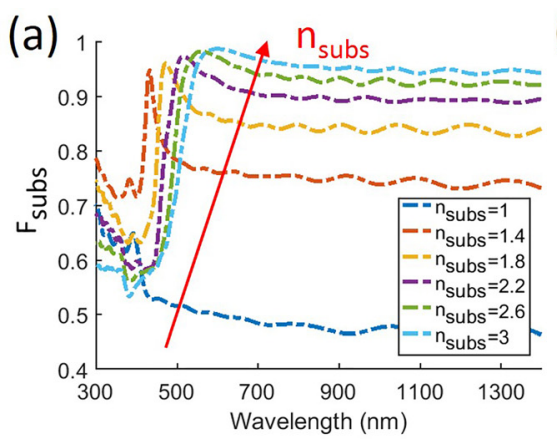

(b)

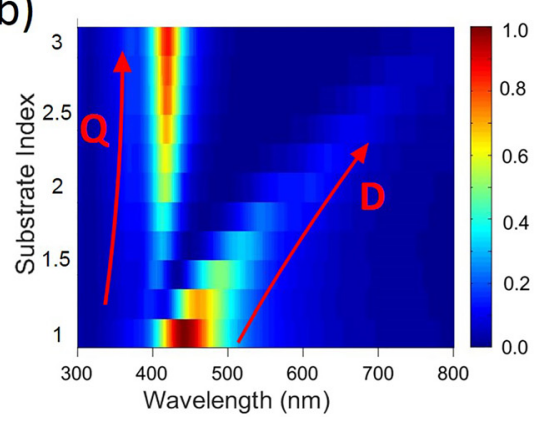

(c)

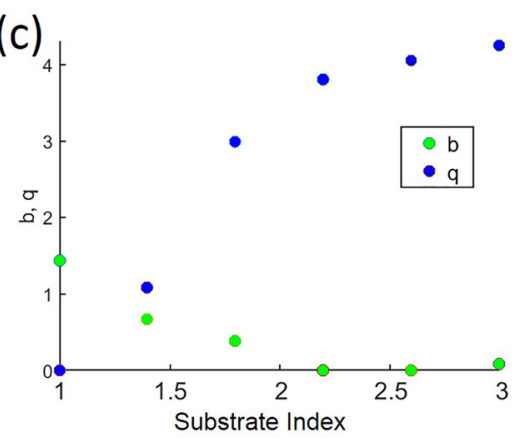

FIG. 2. (a) Fractional scattered forward and (b) backscattering for $75 \mathrm{~nm}$ width, $5 \mathrm{~nm}$ RoC cubic Ag NPs above a bare substrate with varying refractive index. (c) The relationship between the asymmetry parameter $q$ and the resonance modulation parameter $b$ from Eq. (2) to $\mathrm{n}_{\text {subs }}$. 


$$
R(\omega)=\sigma_{s}(\omega) \sigma_{a}(\omega)
$$

where

$$
\sigma_{a}(\omega)=\frac{\left(\frac{\omega^{2}-\omega_{a}^{2}}{2 W_{a} \omega_{a}}+q\right)^{2}+b}{\left(\frac{\omega^{2}-\omega_{a}^{2}}{2 W_{a} \omega_{a}}\right)^{2}+1} .
$$

Here, $\omega_{a}$ is the central wavelength associated with the Fano resonance, $W_{a}$ is the resonance width, $q$ is the asymmetry parameter, and $b$ is the modulation damping parameter. The shape of the Fano resonance is defined entirely by the parameters $q$ and $b$ : The damping parameter $b$ is described as the ratio of energy lost in the system as heat to the energy coupled between modes. Weaker coupling between modes or larger thermal losses will therefore produce a higher $b$ value, greater modulation, and a weaker Fano resonance. The asymmetry parameter $q$ can be interpreted as the amplitude of the dark mode relative to the continuum near the Fano resonance, ${ }^{14}$ and is another measure of the strength of the mode coupling. Falling values of $b$ and rising $q$ are both indications of increased mode coupling.

For particles supporting more than two modes, Fano effects can also be observed due to higher order resonances ${ }^{22}$ and the backscattering spectra can be expressed

$$
R(\omega)=\sigma_{s}(\omega) \sigma_{a 1}(\omega) \sigma_{a 2}(\omega) \ldots \ldots \ldots \sigma_{a n}(\omega),
$$

where the asymmetric Fano resonances are labelled as $a 1$, $a 2$, etc. In Fig. 1(c), it can be seen that there is a small third mode at around $365 \mathrm{~nm}$, which has previously been attributed to higher order oscillations about the face of the cube. ${ }^{35}$ To obtain the best fits, this must be taken into account, and so, Eq. (3) is the form used to obtain the fitting results in this investigation.

Figure 2(c) shows the impact of increasing $\mathrm{n}_{\text {subs }}$ on these two parameters. Eq. (3) is fitted to the backscatter plots in Fig. 2(b) to determine the parameters $b$ and $q$ for changing the substrate index. The modulation damping parameter $b$ is seen to decline as $\mathrm{n}_{\text {subs }}$ is raised, which represents a stronger coupling between modes and fits well with strong intermodal interactions associated with the hybridisation model described earlier. Higher index materials polarise more strongly and so as $\mathrm{n}_{\text {subs }}$ is raised the symmetry breaking about the lower face becomes more significant, which enables greater interaction between the modes and thus a more pronounced Fano resonance. The reduction in $b$ is also responsible for the change in the shape of the Fano effect in Fig. 2(a), from displaying a peak for $\mathrm{n}_{\text {subs }}=1.4$ to a strong dip at higher substrate indexes. ${ }^{13} q$ is seen to increase with $\mathrm{n}_{\text {subs }}$, due to the hybridisation also strengthening the backscattering of the peak corresponding to the upper mode.

Thus, the RI of the substrate is observed to have two different effects. The first is that the higher optical density of states encourages stronger forward coupling from dipolar scatterers and produces better forward coupling across most of the spectrum. This is the reason that plasmonic scatterers continue to be such an attractive potential addition to devices with high-index substrates such as $\mathrm{Si}$ and GaAs. The converse of this however is that a high RI substrate produces a strong interaction between modes, leading to significant hybridisation and mode splitting. This leads to a strong increase in the Fano resonance and a greater drop in forward scattering at low frequencies.

Whilst this study is focussed on nanocubes, it should be noted that these trends will apply to any flat-bottomed NPs, although the specific particle geometry will define the strength and spectra of the modes and therefore the strength of the Fano interaction for any given environment. Rounded particles such as spheres do not generally interact strongly enough with a substrate to produce hybridisation unless light is incident from oblique angles, ${ }^{36}$ and so are outside the scope of this investigation. However, the addition of a thin film has also been shown to produce broadband enhancements in forward coupling for spheres. ${ }^{37}$

Since it is the strong RI gradient at the substrate-air interface enabling the mode interaction that produces the strong Fano resonances, it follows that the Fano effect can be suppressed by reducing this contrast. The most straightforward method to achieve this is to simply place the NP in an upper medium with a greater RI than that of free space, as shown in Fig. 3. This is a useful observation, as more often than not in a completed device the active layer will not be exposed to air and will often be covered by a glass layer or overcoating protective film.

The effect of altering the upper layer index $\mathrm{n}_{\text {air }}$ above an NP on a substrate where $\mathrm{n}_{\text {subs }}=2$ can be clearly seen in Figure 3. In Fig. 3(a), the forward coupling across the majority of the spectrum is seen to be reduced with rising $\mathrm{n}_{\text {air }}$ due to the reduction in the difference in DoS between the layers.
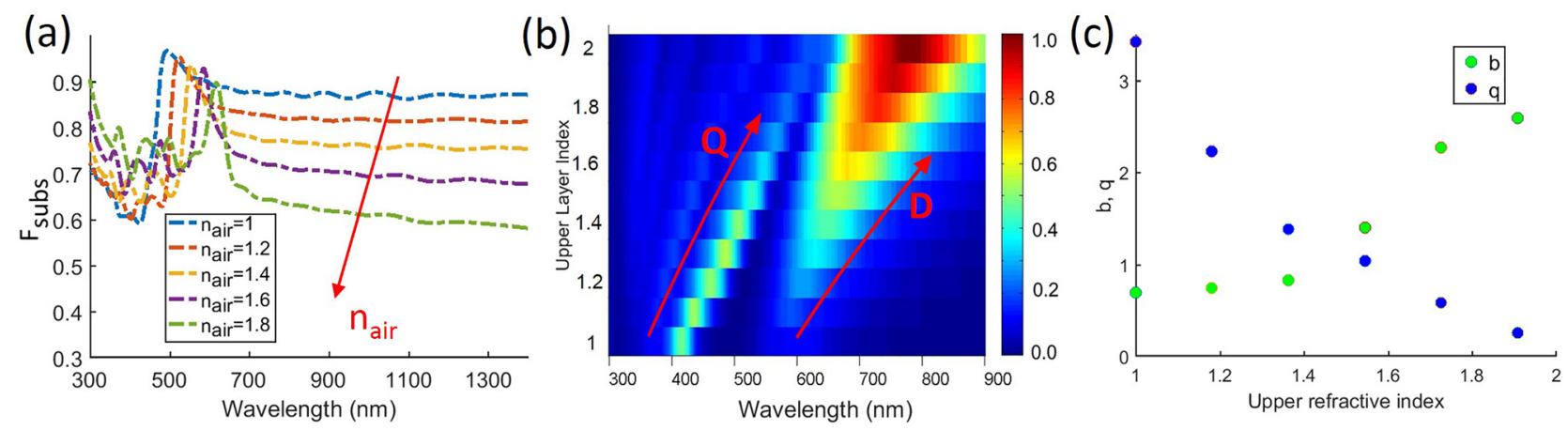

FIG. 3. (a) $\mathrm{F}_{\text {subs }}$ and (b) backscattering for $75 \mathrm{~nm}$ width, $5 \mathrm{~nm}$ RoC cubic Ag NPs above a bare substrate with $\mathrm{n}_{\text {subs }}=2$ and varying $\mathrm{n}_{\text {air }}$. (c) The relationship between $q, b$ from Eq. (2), and $\mathrm{n}_{\text {air }}$. 
The modes are also seen to redshift, and additional modes are seen to appear at the short wavelength end of the spectrum. This can be observed more clearly in Fig. 3(b), where it can also be seen that the $\mathbf{Q}$ mode, concentrated about the upper face of the NP is affected much more strongly by the index of the upper layer since the lower face $\mathbf{D}$ mode principally interacts with the substrate. In Fig. 3(a), it can also be seen that the Fano drop is much reduced. This can be explained by the hybridisation model described earlier, and fitting the backscatter curves to Eq. (3); in Fig. 3(c), it can be observed that as $\mathrm{n}_{\text {air }}$ is raised, the asymmetry parameter $q$ is reduced and the modulation damping parameter $b$ increases, indicating a weakened interaction between modes with greater $\mathrm{n}_{\text {air }}$. This further supports the idea that it is the increased hybridisation of modes in the NP that leads to the increased Fano response.

By changing the RI of the upper layer then, it is possible to reduce the mode hybridisation and therefore minimise the Fano losses in forward scattering at short wavelengths. However, this comes at the cost of drastic reduction in forward coupling across the majority of the spectrum, as there is a reduced DoS difference between layers and the $\mathbf{D}$ mode decouples from the substrate, so any gains at short wavelengths are rendered insignificant.

Another common way to control NP resonances for light trapping is to introduce a spacer layer between the particle and the substrate (see Fig. 1(a)). ${ }^{23,38,39}$ This is discussed further in the supplementary material, but since this technique also relies solely upon weakening the interaction between the excited modes and a high index substrate by reducing the RI gradient, the results are largely similar to the above and result in a marked decrease in $\mathrm{F}_{\text {subs }}$ with the spacer thickness.

A method which has been suggested to control scattering in planar devices is to overcoat the NP with a thin dielectric film with an RI such that $\mathrm{n}_{\text {air }}<\mathrm{n}_{\text {film }}<\mathrm{n}_{\text {subs. }}{ }^{37,40,41}$ In this scenario, light initially back scattered at an angle larger than the critical angle for the film-air interface is reflected forward into the substrate via total internal reflection, which can lead to significant improvements in the forward scattering. This has been demonstrated generally, ${ }^{37}$ and through incorporating the scattering particles into the $\mathrm{SiN}$ antireflection coating of a silicon solar cell. ${ }^{40,41}$ This is shown in electric field plots of the scattered field from an NP at a bare interface where $\mathrm{n}_{\text {subs }}=2$, and with an overcoating film with $\mathrm{n}_{\text {film }}=1.5$ in Figs. 4(a) and 4(b). In Fig. 4(b), backscattered light is initially coupled into the quasiwaveguide modes of the film, and then can be seen to leak out into the substrate, resulting in a significant reduction in light escaping away from the substrate.

As the RI of the film, $\mathrm{n}_{\text {flm }}$ is raised, for a film that fully covers the NPs near field $(300 \mathrm{~nm}$ thick films were used here, although the thickness has been shown to have a negligible impact on $\mathrm{F}_{\text {subs }}$ for thin films $^{37}$ ), the modes are altered in exactly the same way as described in Fig. 3. Thus, raising $\mathrm{n}_{\text {fim }}$ can dramatically reduce the Fano drop as with $\mathrm{n}_{\text {air }}$. The expected drop in forward scattering associated with this seen in Fig. 3 is not observed however, since raising the RI of the film also changes the reflection conditions with the air layer: most importantly, it reduces the angular escape cone; therefore, the
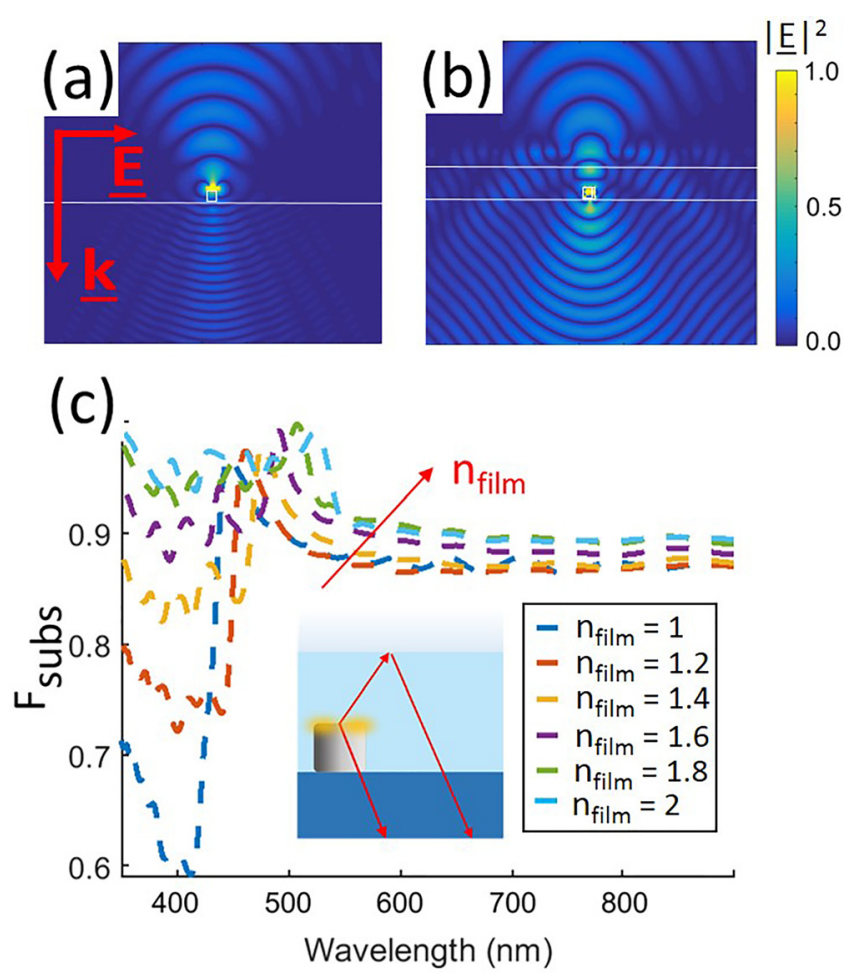

FIG. 4. (a) and (b) The electric fields of light scattered at $400 \mathrm{~nm}$ from an $\mathrm{NP}$ on a bare substrate with $\mathrm{n}_{\text {subs }}=2$ and one with an $300 \mathrm{~nm}$ overcoating film with $\mathrm{n}_{\text {film }}=1.5$, highlighting the effect of the film to enhance forward scattering. (c) $\mathrm{F}_{\text {subs }}$ for a $75 \mathrm{~nm}$ width, $5 \mathrm{~nm}$ RoC Ag cubic NP overcoated with a $300 \mathrm{~nm}$ film with varying RI, $\mathrm{n}_{\text {film }}$. Inset: the geometry used for the simulations showing the layers and the reflection of scattered light from the film interface, leading to enhanced forward scatter.

initially backscattered light is totally internally reflected at the film-air interface and greater coupling is achieved across the solar spectrum. This is demonstrated in Fig. 4(c) where it can be seen that the Fano drop can be totally removed by the film whilst the forward coupling is improved on the case of a particle at a bare interface across the spectrum.

The aim of this letter was to reduce short wavelength backscatter for particles above a high index substrate, which has been detrimental to progress in the field of plasmonic light trapping for nearly two decades. It was found that the Fano resonance producing this switch in directionality was strengthened for NPs near high-index substrates due to the polarisation of the substrate inducing coupling between modes. When results from simulations are fitted to the model of Gallinet and Martin, the behaviour of the screening and asymmetry parameters with $\mathrm{n}_{\text {subs }}$ help provide a detailed description of the impact of the substrate on mode coupling and forward scattering from individual nanoparticles.

It was found that the Fano response could be muted by raising the index of the layer above the particle or by separating the NP from the substrate by a thin spacer in order to reduce the RI gradient and hence the mode interaction which produces the strong Fano effect. However, both of these methods also lead to reduced forward scattering across most of the spectrum due to reducing the coupling of scattered light to the substrate. Adding a thin film above the NP thick enough to decouple the particles near field from the upper layer led to an equivalent reduction in mode hybridisation and subsequent reduction of the Fano resonance. In this case, 
reflection from the film-air boundary leads to an increased forward scatter across the solar spectrum and provides an elegant solution to the issue of Fano losses in plasmonic light trapping.

See supplementary material for details on simulations used, further explanations of the hybrid modes in flatbottomed particles, and simulations of spacer layer effects.

A.W.P. acknowledges support from the U.K. Engineering and Physical Sciences Research Council.

${ }^{1}$ M. Garcia, J. Phys. D: Appl. Phys. 44, 283001 (2011).

${ }^{2}$ K. M. A. El-Nour, A. Eftaiha, A. Al-Warthan, and R. A. Amma, Arabian J. Chem. 3(3), 135-140 (2010)

${ }^{3}$ G. M. Akselrod, C. Argyropoulos, T. B. Hoang, C. Ciracì, C. Fang, J. Huang, D. R. Smith, and M. H. Mikkelsen, Nat. Photonics 8, 835-840 (2014).

${ }^{4}$ A. Powell, N. Hjerrild, A. Watt, H. Assender, and J. Smith, Appl. Phys. Lett. 104(8), 081110 (2014).

${ }^{5}$ A. G. Curto, G. Volpe, T. H. Taminiau, M. P. Kreuzer, R. Quidant, and N. F. van Hulst, Science 329(5994), 930-933 (2010).

${ }^{6} \mathrm{P}$. Wang, L. Zhang, Y. Xia, L. Tong, X. Xu, and Y. Ying, Nano Lett. 12(6), 3145-3150 (2012).

${ }^{7}$ A. W. Powell, D. M. Coles, R. A. Taylor, A. A. R. Watt, H. E. Assender, and J. M. Smith, Adv. Opt. Mater. 4(4), 634-642 (2016).

${ }^{8}$ N. Liu, M. L. Tang, M. Hentschel, H. Giessen, and A. P. Alivisatos, Nat. Mater. 10(8), 631-636 (2011).

${ }^{9}$ M. Gu, Z. Ouyang, B. Jia, N. Stokes, X. Chen, N. Fahim, X. Li, M. J. Ventura, and Z. Shi, Nanophotonics 1(3-4), 235-248 (2012).

${ }^{10}$ H. Atwater and P. Polman, Nat. Mater. 9, 205-213 (2010).

${ }^{11}$ K. R. Catchpole and A. Polman, Appl. Phys. Lett. 93, 191113 (2008).

${ }^{12}$ V. Ferry, J. N. Munday, and H. A. Atwater, Adv. Mat. 22(43), 4794-4808 (2010).

${ }^{13}$ B. Gallinet and O. J. Martin, Phys. Rev. B 83(23), 235427 (2011).

${ }^{14}$ B. Gallinet and O. J. Martin, ACS Nano 5(11), 8999-9008 (2011).

${ }^{15}$ S. Zhang, K. Bao, N. J. Halas, H. Xu, and P. Nordlander, Nano Lett. 11(4), 1657-1663 (2011).

${ }^{16}$ F. Liu and J. Jin, J. Opt. 17(5), 055004 (2015).

${ }^{17}$ N. S. King, L. Liu, X. Yang, B. Cerjan, H. O. Everitt, P. Nordlander, and N. J. Halas, ACS Nano 9(11), 10628-10636 (2015).
${ }^{18}$ Y. Zhang, F. Wen, Y.-R. Zhen, P. Nordlander, and N. J. Halas, Proc. Natl. Acad. Sci. U.S.A. 110(23), 9215-9219 (2013).

${ }^{19}$ G. F. Walsh and L. D. Negro, Nano Lett. 13(7), 3111-3117 (2013).

${ }^{20}$ Z.-J. Yang, Z.-S. Zhang, Z.-H. Hao, and Q.-Q. Wang, Appl. Phys. Lett. 99(8), 081107 (2011).

${ }^{21}$ A. Ridolfo, O. Di Stefano, N. Fina, R. Saija, and S. Savasta, Phys. Rev. Lett. 105(26), 263601 (2010).

${ }^{22}$ B. Luk'yanchuk, N. I. Zheludev, S. A. Maier, N. J. Halas, P. Nordlander, H. Giessen, and C. T. Chong, Nat. Mater. 9, 707-715 (2010).

${ }^{23}$ P. Spinelli, C. van Lare, E. Verhagen, and A. Polman, Opt. Express 19, A303-A311 (2011).

${ }^{24}$ H. Chen, L. Shao, T. Ming, K. C. Woo, Y. C. Man, J. Wang, and H.-Q. Lin, ACS Nano 5(8), 6754-6763 (2011).

${ }^{25}$ P. Nordlander and E. Prodan, Nano Lett. 4(11), 2209-2213 (2004).

${ }^{26}$ R. Nicolas, G. Lévêque, J. Marae-Djouda, G. Montay, Y. Madi, J. Plain, Z. Herro, M. Kazan, P.-M. Adam, and T. Maurer, Sci. Rep. 5, 14419 (2015).

${ }^{27}$ Z. Starowicz, G. Kulesza-Matlak, and M. Lipiński, Plasmonics 10(6), 1639-1647 (2015).

${ }^{28}$ S. H. Lim, W. Mar, P. Matheu, D. Derkacs, and E. T. Yu, J. Appl. Phys. 101, 104309 (2007).

${ }^{29}$ Y. A. Akimov and W. S. Koh, Plasmonics 6(1), 155-161 (2010).

${ }^{30}$ F. J. Beck, E. Verhagen, S. Mokkapati, A. Polman, and K. R. Catchpole, Opt. Express 19, A146-A156 (2011).

${ }^{31}$ J. M. McMahon, Y. Wang, L. J. Sherry, R. P. Van Duyne, L. D. Marks, S. K. Gray, and G. C. Schatz, J. Phys. Chem. C 113(7), 2731-2735 (2009).

${ }^{32}$ J. van de Groep, D. Gupta, M. A. Verschuuren, M. M. Wienk, R. A. Janssen, and A. Polman, Sci. Rep. 5, 11414 (2015).

${ }^{33}$ See http://nanocomposix.com/ for Nanocomposix, 2016.

${ }^{34}$ See http://www.lumerical.com/ for Lumerical-Solutions, 2016.

${ }^{35}$ J. B. Lassiter, F. McGuire, J. J. Mock, C. Cirac, R. T. Hill, B. J. Wiley, A. Chilkoti, and D. R. Smith, Nano Lett. 13(12), 5866-5872 (2013).

${ }^{36}$ M. W. Knight, Y. Wu, J. B. Lassiter, P. Nordlander, and N. J. Halas, Nano Lett. 9(5), 2188 (2009).

${ }^{37}$ A. W. Powell, M. B. Wincott, A. A. R. Watt, H. E. Assender, and J. M. Smith, J. Appl. Phys. 113(18), 184311 (2013).

${ }^{38}$ S. Zeng, X. Yu, W.-C. Law, Y. Zhang, R. Hu, X.-Q. Dinh, H.-P. Ho, and K.-T. Yong, Sens. Actuators, B 176, 1128-1133 (2013).

${ }^{39}$ S. Pillai, F. J. Beck, K. R. Catchpole, Z. Ouyang, and M. A. Green, J. Appl. Phys. 109, 073105 (2011).

${ }^{40}$ N. F. Fahim, Z. Ouyang, B. Jia, Y. Zhang, Z. Shi, and M. Gu, Appl. Phys. Lett. 101, 261102 (2012).

${ }^{41}$ S. Xie, Z. Ouyang, N. Stokes, B. Jia, and M. Gu, J. Appl. Phys. 115(19), 193102 (2014). 